\title{
Characteristics and risk factors associated with critical illness in pediatric COVID-19
}

\author{
Grace Fisler ${ }^{1,2^{*}} \mathbb{D}$, Stephanie M. Izard ${ }^{3}$, Sareen Shah ${ }^{1,2}$, Deirdre Lewis ${ }^{1,2}$, Mundeep K. Kainth ${ }^{2,4}$, \\ Stefan H. F. Hagmann 2,4 , Joshua A. Belfer ${ }^{2}$, Lance M. Feld ${ }^{2}$, Fiore Mastroianni ${ }^{5}$, Charlotte L. Kvasnovsky ${ }^{2,6}$, \\ Christine A. Capone ${ }^{1,2}$, James Schneider ${ }^{1,2}$, Todd Sweberg ${ }^{1,2}$, Charles Schleien 1,2, Matthew D. Taylor ${ }^{1,2}$ and the \\ Northwell COVID-19 Research Consortium
}

\begin{abstract}
Background: While much has been reported regarding the clinical course of COVID-19 in children, little is known regarding factors associated with organ dysfunction in pediatric COVID-19. We describe critical illness in pediatric patients with active COVID-19 and identify factors associated with PICU admission and organ dysfunction. This is a retrospective chart review of 77 pediatric patients age 1 day to 21 years admitted to two New York City pediatric hospitals within the Northwell Health system between February 1 and April 24, 2020 with PCR +SARS-CoV-2. Descriptive statistics were used to describe the hospital course and laboratory results and bivariate comparisons were performed on variables to determine differences.

Results: Forty-seven patients (61\%) were admitted to the general pediatric floor and thirty (39\%) to the PICU. The majority $(97 \%, n=75)$ survived to discharge, $1.3 \%(n=1)$ remain admitted, and $1.3 \%(n=1)$ died. Common indications for PICU admission included hypoxia (50\%), hemodynamic instability (20\%), diabetic ketoacidosis (6.7\%), mediastinal mass (6.7\%), apnea (6.7\%), acute chest syndrome in sickle cell disease (6.7\%), and cardiac dysfunction (6.7\%). Of PICU patients, $46.7 \%$ experienced any significant organ dysfunction (pSOFA $>=2$ ) during admission. Patients aged 12 years or greater were more likely to be admitted to a PICU compared to younger patients $(p=0.015)$. Presence of an underlying comorbidity was not associated with need for PICU admission $(p=0.227)$ or organ dysfunction $(p=0.87)$. Initial white blood cell count (WBC), platelet count, and ferritin were not associated with need for PICU admission. Initial C-reactive protein was associated with both need for PICU admission $(p=0.005)$ and presence of organ dysfunction $(p=0.001)$. Initial WBC and presenting thrombocytopenia were associated with organ dysfunction ( $p=0.034$ and $p=0.003$, respectively).
\end{abstract}

Conclusions: Age over 12 years and initial CRP were associated with need for PICU admission in COVID-19. Organ dysfunction was associated with elevated admission CRP, elevated WBC, and thrombocytopenia. These factors may be useful in determining risk for critical illness and organ dysfunction in pediatric COVID-19.

Keywords: COVID-19, CRP, Organ dysfunction, Pediatrics

*Correspondence: gfisler@northwell.edu

${ }^{1}$ Division of Pediatric Critical Care Medicine, Steven and Alexandra Cohen Children's Medical Center of New York, Northwell Health, Donald and Barbara Zucker School of Medicine At Hofstra/Northwell, 269-01 76th Ave, New Hyde Park, NY 11040, USA

Full list of author information is available at the end of the article

\section{Background}

The SARS-CoV-2 virus has overwhelmed healthcare systems around the world [1,2]. Disease presentation varies from asymptomatic infections identified through contact tracing to fulminant respiratory failure refractory to conventional therapies. Initial data concluded that children experienced predominantly mild illness with only a handful requiring ICU admission and mechanical ventilation 
[3-8]. Later data identified serious disease in a small subset of children, but concluded the vast majority experience mild infection $[9,10]$.

The first study on critically ill children with COVID-19 described 48 patients admitted to 14 pediatric intensive care units (PICU) in the U.S. Over $80 \%$ of patients had underlying medical conditions and two died [11]. These data are congruent with another multicenter study that described a high rate of comorbidities and adolescent age in pediatric patients requiring PICU admission for COVID-19 [12]. Need for mechanical ventilation in children with COVID-19 has been associated with elevated CRP in one single-center study [13].

The clinical course of pediatric COVID-19 has been described in many studies, however there is a paucity of literature examining factors that contribute to organ dysfunction in children [14-16]. In this study, we set out to determine factors associated with PICU admission and acute organ dysfunction in COVID-19 patients. We compare the characteristics between three groups of pediatric patients admitted with COVID-19 infection: patients not requiring PICU care, patients admitted to the PICU with minimal or no organ dysfunction, and patients admitted to the PICU with organ dysfunction.

\section{Materials and methods}

We conducted a retrospective cohort study of all patients under 21 years of age $(n=77)$ admitted with COVID-19 to either of our two hospitals that offer pediatric intensive care from February 1, 2020 through April 24, 2020, during the height of the pandemic in the New York City area. Data collection closed on June 20, 2020. The dataset was the same used in an epidemiology study examining admitting symptoms, but encompassed a longer study period and included patients at our second hospital [17]. The study was approved by the Northwell Health System Institutional Review Board (\#20-0318-CCMC) and granted a waiver of informed consent.

Cases were identified by medical record documentation of COVID-19 infection in any patient admitted to our pediatrics service. Data collected included demographics, indication for admission, BMI, presence of preexisting conditions, hospital outcomes, and PELOD-2 and pSOFA scores $[18,19]$. Lab values were collected within $24 \mathrm{~h}$ of admission and included white blood cell count (WBC), platelet count, C-reactive protein (CRP), and ferritin. ICU interventions were also recorded and analyzed. Pediatric acute respiratory distress syndrome (pARDS) was defined using non-invasive measurements of oxygenation [20].

Data were collected and managed using REDCap electronic data capture tools hosted at Northwell
Health [21, 22]. All analyses were performed using SAS Studio version 3.8 with a $p$ value $<0.05$ considered statistically significant.

We identified several possible factors associated with ICU admission and organ dysfunction a priori. These were extrapolated from adult COVID-19 and pediatric multiorgan dysfunction literature. Factors suspected to be associated with severe disease included older age, presence of pre-existing comorbidity, increased BMI, initial WBC, platelet count, CRP, and ferritin [23-25].

Age was categorized into $<60$ days, 60 days to $<5$ years, 5 to $<12$ years, or $>=12$ years, based on sample distribution and clinical relevance. For inferential analysis, age categories were collapsed into $<12$ years versus $>=12$ years. BMI was converted into BMI percentile for those $<20$ years old [26]. Patients in $<5$ th percentile were categorized as underweight, 5-85th as normal weight, 85-95th as overweight, and $>95$ th as obese. For patients aged 20-21 years, those with $\mathrm{BMI}<18.5$ were considered underweight, 18.5 to 25 as normal weight, 25 to 30 as overweight, and $>30$ as obese[27]. Patients $<2$ years were excluded in BMI categorization. For these analyses, categorized BMI was dichotomized into (1) underweight or normal weight and (2) overweight or obese. Need for respiratory support was defined by need for high-flow nasal cannula (HFNC) above $4 \mathrm{~L}$ per minute, non-invasive positive pressure ventilation (NIPPV), or invasive mechanical ventilation (MV). Patients with missing data were excluded from individual inferential analyses. Outcomes of interest included ICU admission during hospital stay (yes versus no) and organ dysfunction (yes versus no).

All variables were summarized descriptively within the overall sample and the subgroups of floor only, ICU admission for organ dysfunction and for other reasons. The Chi-square or Fisher's exact test was used for categorical variables of interest such as any significant differences across ICU admission and organ dysfunction outcomes. The Wilcoxon rank sum test was used for continuous variables.

The pSOFA score is an adaptation of the adult sequential organ function assessment (SOFA), a part of the current adult definition of sepsis $[18,28]$. An increase in SOFA score of $>=2$ is associated with a mortality of $10 \%$ in adult patients [29]. We utilized an increase in PSOFA score of $>=2$ to define significant organ dysfunction in pediatric patients. We screened for baseline organ dysfunction when information was available and adjusted the pSOFA score to reflect the increase in PSOFA score from baseline. We also examined the PELOD-2 (Pediatric Logistic Organ Dysfunction) score as a second measure. 


\section{Results}

General characteristics of patients admitted for pediatric COVID-19 (Table 1)

Seventy-seven patients met the inclusion criteria.

All patients were COVID-19 PCR positive by nasopharyngeal swab. All included patients had primary COVID-19 infection and none were diagnosed with Multisystem Inflammatory Syndrome in Children (MIS-

C) as the data collection period encompassed the time of the initial, primary outbreak of COVID-19 in the region. Of 77 patients (mean age $=9.5$ years), 71 were admitted to the tertiary children's medical center and six to the satellite hospital. Thirty (39\%) were admitted to a PICU and $47(61 \%)$ to a general inpatient ward. No differences were noted in gender, race, ethnicity, or general preexisting comorbidities.

Table 1 Demographic data and comorbidities

\begin{tabular}{|c|c|c|c|c|}
\hline & Total cohort $(N=77)$ & Floor patients $(N=47)$ & $\begin{array}{l}\text { PICU low acuity } \\
(n=16)^{\mathrm{a}}\end{array}$ & $\begin{array}{l}\text { PICU organ } \\
\text { dysfunction } \\
(n=14)^{b}\end{array}$ \\
\hline \multicolumn{5}{|l|}{ Demographic data } \\
\hline \multicolumn{5}{|l|}{ Age $(n, \%)$} \\
\hline Age $<60$ days & $20(26)$ & $16(34)$ & $3(19)$ & $1(7)$ \\
\hline Age 60 days to $<5$ years & $10(13)$ & $6(13)$ & $2(13)$ & $2(14)$ \\
\hline Age 5 years to $<12$ years & $9(12)$ & $7(15)$ & 0 & $2(14)$ \\
\hline Age $>=12$ years & $38(49)$ & $18(38)$ & $11(69)$ & $9(64)$ \\
\hline Female sex $(n, \%)$ & $40(53)$ & $23(49)$ & $9(56)$ & $8(57)$ \\
\hline \multicolumn{5}{|l|}{ BMI (percentile, $n, \%)^{\mathrm{c}, \mathrm{d}}$} \\
\hline Underweight (<5th percentile) & $2(4)$ & $2(8)$ & 0 & 0 \\
\hline Normal weight (5-85th percentile) & $26(58)$ & $13(52)$ & $7(78)$ & $6(55)$ \\
\hline Overweight (85-95th percentile) & $5(11)$ & $3(12)$ & $1(11)$ & $1(9)$ \\
\hline Obese (>95th percentile) & $12(27)$ & $7(28)$ & $1(11)$ & $4(36)$ \\
\hline \multicolumn{5}{|l|}{ Race $(n, \%)$} \\
\hline Black & $22(29)$ & $10(21)$ & $7(44)$ & $5(38)$ \\
\hline White & $15(19)$ & $12(26)$ & $3(19)$ & 0 \\
\hline Asian & $9(12)$ & $5(11)$ & $1(6)$ & $3(21)$ \\
\hline Multiracial/other & $28(36)$ & $17(36)$ & $5(31)$ & $6(43)$ \\
\hline Unknown & $3(4)$ & $3(6)$ & 0 & 0 \\
\hline \multicolumn{5}{|l|}{ Ethnicity $(n, \%)$} \\
\hline Hispanic & $18(23)$ & $9(19)$ & $3(19)$ & $6(43)$ \\
\hline Non-Hispanic & $55(71)$ & $37(79)$ & $11(69)$ & $7(50)$ \\
\hline Unknown & $4(5)$ & $1(2)$ & $2(13)$ & $1(7)$ \\
\hline \multicolumn{5}{|l|}{ Comorbidities $(n, \%)$} \\
\hline Prematurity & $7(9)$ & $6(13)$ & $1(6)$ & 0 \\
\hline Respiratory disease & 16 & $8(17)$ & 5 & $3(21)$ \\
\hline Congenital heart disease & $5(6)$ & $2(4)$ & $2(13)$ & $1(7)$ \\
\hline Diabetes mellitus & $5(6)$ & $2(4)$ & $2(13)$ & $1(7)$ \\
\hline Immunosuppression & $9(12)$ & $6(13)$ & $2(13)$ & $1(7)$ \\
\hline Autoimmune disorder & $2(3)$ & $1(2)$ & $1(6)$ & 0 \\
\hline Bone marrow transplant & $2(3)$ & $1(2)$ & 0 & $1(2)$ \\
\hline Kidney disease & $1(1)$ & $1(2)$ & 0 & 0 \\
\hline Cancer & $5(6)$ & $3(6)$ & $1(6)$ & $1(7)$ \\
\hline Genetic disorder & $7(9)$ & $2(4)$ & $1(6)$ & $4(29)$ \\
\hline
\end{tabular}

a PICU low acuity defined as pSOFA score $<2$

b PICU organ dysfunction defined as pSOFA score $>=2$

c BMI category presented for 45 patients ( 25 floor, 11 ICU organ days, 9 other). 25 missing under 2 years old, 7 missing older than 2 years

d BMl category for those 20-21 classified as underweight $<18.5$, normal 18.5-25, overweight 25-30, obese $>30$ 


\section{Characterization of organ dysfunction associated with pediatric COVID-19}

Of 30 ICU patients, $50 \%$ presented with hypoxia and $20 \%$ with hemodynamic instability. Other notable admission indications are in Table 2. Within \pm four hours of PICU admission, $63.3 \%$ experienced documented hypoxia (defined as $\mathrm{SpO} 2<90 \%$ on room air) and $10 \%$ required vasoactive medications. Two patients $(6.7 \%$ of PICU cohort) required renal replacement therapy. There were no patients with underlying chronic kidney disease in the PICU cohorts. Of PICU patients, $46.7 \%$ experienced organ dysfunction (Table 2). Specific organ system dysfunction, defined using pSOFA score $>=1$ for organ system category, showed a predominance of cardiovascular and respiratory failure (Table 2).

\section{Characterization of respiratory failure (Table 2)}

Among patients admitted to the PICU, NIPPV (bilevel or continuous positive pressure, HFNC) was required by $30 \%$ of patients; $6.7 \%$ were managed exclusively with HFNC.

Among PICU patients, 27\% $(n=8)$ required endotracheal intubation. The average oxygen saturation index (OSI), a non-invasive measure of lung disease severity, upon intubation was 14. Median number of ventilatorfree days was 15 . One patient required veno-venous extracorporeal membranous oxygenation (4 days) for severe pARDS, and one patient was escalated to highfrequency percussive ventilation and eventually died secondary to refractory hypoventilation with severe hypercarbia and hypoxemia. This patient was evaluated for ECMO and deemed not to be a candidate based on

Table 2 PICU admittance and characteristics

\begin{tabular}{|c|c|c|c|}
\hline \multicolumn{3}{|c|}{ Reason(s) for PICU admission $n$ (\%) } & Total PICU cohort $(N=30)$ \\
\hline \multicolumn{3}{|l|}{ Hypoxia } & $15(50)$ \\
\hline \multicolumn{3}{|l|}{ Hemodynamic instability } & $6(20)$ \\
\hline \multicolumn{3}{|l|}{ DKA } & $2(7)$ \\
\hline \multicolumn{3}{|l|}{ Cardiac dysfunction } & $2(7)$ \\
\hline \multicolumn{3}{|l|}{ Mediastinal mass } & $2(7)$ \\
\hline \multicolumn{3}{|l|}{ Apnea } & $2(7)$ \\
\hline \multicolumn{3}{|c|}{ Acute chest syndrome in sickle cell disease } & $2(7)$ \\
\hline \multicolumn{3}{|c|}{ Other } & $6(20)$ \\
\hline \multicolumn{4}{|l|}{ Outcomes } \\
\hline \multicolumn{3}{|c|}{ Length of ICU stay, days [mean (median, IQR)] } & $8.8(3.6,2.5-14.7)$ \\
\hline \multicolumn{3}{|c|}{ ICU discharge $(n, \%)$} & $29(97)$ \\
\hline \multicolumn{3}{|l|}{ Deceased $(n, \%)$} & $1(3)$ \\
\hline \multicolumn{3}{|l|}{ Respiratory failure } & Intubated cohort $(N=8)$ \\
\hline \multicolumn{3}{|c|}{ OSI at intubation [mean (median, IQR)] } & $14(15,5.5-20)$ \\
\hline \multicolumn{3}{|c|}{ Maximum PEEP [mean (median)] } & $12(13.5)$ \\
\hline \multicolumn{3}{|c|}{ Ventilator-free days [mean (median, IQR)] } & $14(15,13-23)$ \\
\hline PICU admission characteristics & Total cohort $(N=30)$ & PICU low acuity $(n=16)$ & $\begin{array}{l}\text { PICU organ } \\
\text { dysfunction } \\
(n=14)\end{array}$ \\
\hline pSOFA [mean (median, IQR)] & $2.3(1.0,1.0-3.0)$ & $0.6(1.0,0-1.0)$ & $44(3.0,2.0-7.0)$ \\
\hline PELOD-2 [mean (median, IQR)] & $2.0(2.0,0-3.0)$ & $0.6(0,0-2.0)$ & $3.6(3.5,2.0-6.0)$ \\
\hline \multicolumn{4}{|l|}{ System failure $(n, \%)^{\mathrm{b}}$} \\
\hline Cardiovascular dysfunction & $14(47)$ & $3(19)$ & $11(79)$ \\
\hline Respiratory dysfunction & $9(30)$ & 0 & $9(64)$ \\
\hline Hematalogic dysfunction & $6(20)$ & 0 & $6(43)$ \\
\hline Neurologic dysfunction & $5(17)$ & 0 & $5(36)$ \\
\hline Hepatic dysfunction & $5(17)$ & $2(13)$ & $3(21)$ \\
\hline Renal dysfunction & $5(17)$ & 0 & $5(36)$ \\
\hline
\end{tabular}

${ }^{a}$ Among survivors

b System failure defined as pSOFA $>=1$ for system category(18) 
institutional guidelines due to pre-existing comorbidities, irrespective of COVID-19 status.

\section{Complications, outcomes, and therapies (Table 2)}

Two patients experienced secondary infections during their hospital course (peritonitis and bacterial pneumonia). No concurrent viral infections were documented in any patients.

For those discharged from the PICU $(n=29)$, median PICU length of stay was 3.6 days. At the close of data collection, $98 \%$ of the total cohort was discharged home and one child died (1.3\%).

Interventions targeting COVID-19 among PICU patients included: hydroxychloroquine 63\%, azithromycin $37 \%$, corticosteroids $30 \%$, remdesivir $27 \%$, tocilizumab (anti-IL6R) 13\%, anakinra (anti-IL1R) 13\%, and intravenous immunoglobulin (IVIg) $10 \%$.

\section{Factors associated with PICU admission and organ dysfunction (Table 3)}

Age $\geq 12$ years was associated with the need for PICU admission $(p=0.015)$. Presence of any collected comorbidity was not associated with PICU admission $(p=0.227)$.

Median initial CRP value for floor patients was $9.30 \mathrm{mg} / \mathrm{L}$ and for PICU patients $54.1 \mathrm{mg} / \mathrm{L} \quad(n=37$ patients, $p=0.005)$. Median initial WBC, platelet, and ferritin value showed no difference between floor and PICU patients.

Fourteen patients met criteria for organ dysfunction. Older age, underlying comorbidity, and BMI were not associated with organ dysfunction. Median initial WBC was higher in the organ dysfunction cohort (15.7 versus $7.8 \mathrm{~K} / \mu \mathrm{L}, p=0.034)$. Median initial platelet count was lower in the organ dysfunction cohort (149 versus $268 \mathrm{~K} /$ $\mu \mathrm{L}, p=0.003)$; this association remained when analyzed for non-hematologic organ dysfunction. Median initial ferritin was similar in organ dysfunction patients (454.6 $\mathrm{ng} / \mathrm{mL})$ and non-organ dysfunction patients (487.1 $\mathrm{ng} / \mathrm{mL}$ ). Median initial CRP was higher in the organ dysfunction group than non-organ dysfunction group (109.7 versus $13.9 \mathrm{mg} / \mathrm{L}, p=0.001$ ).

\section{Discussion}

The incidence of critical illness from COVID-19 and risk factors for severe disease in pediatrics are not yet well defined. Multicenter studies have described high rates of comorbid conditions in hospitalized children with COVID-19 [11, 12]. We studied 77 children with COVID19 admitted to two of our hospitals over a 7-week period with the goal of determining clinical characteristics and factors associated with PICU admission and acute organ dysfunction.

In our cohort of pediatric COVID-19, 39\% required intensive care and 18\% developed organ dysfunction, indicating that symptomatic pediatric patients are at risk of critical illness. The most common indications for PICU admission were hypoxia and hemodynamic instability.

Our study found that age $>12$ years is associated with PICU admission $(p=0.015)$ when compared to younger children, although this association was unadjusted. Comorbid pulmonary disease and immunocompromised status have been associated with more severe disease in pediatric patients with other strains of Coronaviridae [30]. Presence of any comorbidity and underlying obesity were not associated with the need for PICU admission or organ dysfunction. The rate of comorbidity in our PICU cohort (42.6\%) and our organ dysfunction cohort (50\%) were notably lower than two previously published studies that utilized reports of disease from many different sites $[11,12]$. As all of our subjects are from the same health system, we may have avoided some testing and reporting bias that may have affected previous multicenter studies, as all patients were triaged and treated with the same protocols. Further, our data indicate that healthy children can suffer severe manifestations of COVID-19 at similar rates to those with chronic medical conditions. There is a need for further study regarding the impact of comorbidities on disease severity in pediatric COVID-19 and regarding the prevalence of symptomatic disease in pediatric populations.

Marked elevations in serum CRP and WBC were noted in our PICU cohort. Elevations in CRP may be predictive of mortality in pediatric severe sepsis and septic shock [24]. Elevated CRP was associated with mortality in an adult COVID-19 cohort from China and need for MV in a pediatric COVID-19 cohort [13, 23]. We identified WBC, platelet count, CRP and ferritin a priori as variables to examine in relation to need for PICU admission and development of organ dysfunction. PICU admission and organ dysfunction were both associated with increased CRP on hospital presentation. Elevated WBC and thrombocytopenia were associated with organ dysfunction in our cohort. Future research to clarify the predictive value of CRP, WBC, and thrombocytopenia is warranted.

Multiple targeted therapies were administered to our cohort of PICU patients. The heterogeneity in treatment approach highlights the need for randomized controlled trials to determine the optimal treatment for the varying phenotypes of COVID-19. Further risk stratification using biomarkers may allow for more efficient trials in the future that can target patients at risk for more severe disease. 
Table 3 Outcomes for PICU admission and organ dysfunction

\begin{tabular}{|c|c|c|c|}
\hline & $\begin{array}{l}\text { PICU admission } \\
N=30\end{array}$ & $\begin{array}{l}\text { No PICU admission } \\
N=47\end{array}$ & $p$ value \\
\hline \multicolumn{4}{|l|}{ Outcome: PICU admission } \\
\hline Age & & & 0.0152 \\
\hline$>=12$ years & $20(66.67)$ & $18(38.30)$ & \\
\hline$<12$ years & $10(33.33)$ & $29(61.70)$ & \\
\hline Any comorbidity & & & 0.2267 \\
\hline Yes & $17(56.67)$ & $20(42.55)$ & \\
\hline No & $13(43.33)$ & $27(57.45)$ & \\
\hline BMI category $(N=45)^{\mathrm{a}}$ & & & 0.7310 \\
\hline Overweight/obese & $7(35.00)$ & $10(40.00)$ & \\
\hline Underweight/normal weight & $13(65.00)$ & $15(60.00)$ & \\
\hline \multicolumn{4}{|l|}{ Laboratory data } \\
\hline Initial CRP $(N=37)$ & & & 0.0053 \\
\hline Median (IQR) & $54.10(13.90,161.90)$ & $9.30(4.00,33.10)$ & \\
\hline Initial ferritin $(N=42)$ & & & 0.8495 \\
\hline Median (IQR) & $446.05(307.35,809.35)$ & $525.30(160.20,1003.00)$ & \\
\hline Initial WBC $(N=69)$ & & & 0.1455 \\
\hline Median (IQR) & $7.8(5.4-17.1)$ & $7.8(4.9-12.3)$ & \\
\hline Initial platelets & & & 0.0612 \\
\hline \multirow[t]{2}{*}{ Median (IQR) } & $231(182-206)$ & $267(200-419)$ & \\
\hline & $\begin{array}{l}\text { Organ dysfunction } \\
N=14\end{array}$ & $\begin{array}{l}\text { No organ dysfunction } \\
N=63\end{array}$ & $p$ value \\
\hline \multicolumn{4}{|l|}{ Outcome: organ dysfunction } \\
\hline Age & & & 0.2166 \\
\hline$>=12$ years & $9(64.29)$ & $29(46.03)$ & \\
\hline$<12$ years & $5(35.71)$ & $34(53.97)$ & \\
\hline Any comorbidity & & & 0.8719 \\
\hline Yes & $7(50.00)$ & $30(47.62)$ & \\
\hline No & $7(50.00)$ & $33(52.38)$ & \\
\hline BMI category $(N=45)^{1}$ & & & 0.7224 \\
\hline Overweight/obese & $5(45.45)$ & $12(35.29)$ & \\
\hline Underweight/normal weight & $6(54.55)$ & $22(64.71)$ & \\
\hline \multicolumn{4}{|l|}{ Laboratory data } \\
\hline Initial CRPC & & & 0.0011 \\
\hline Median (IQR) & $109.70(54.25,218.15)$ & $13.90(4.50,46.70)$ & \\
\hline Initial ferritin ${ }^{b}$ & & & 0.2125 \\
\hline Median (IQR) & $454.60(373.70,1039.00)$ & $487.10(178.90,592.40)$ & \\
\hline Initial WBC $(N=69)$ & & & 0.0341 \\
\hline Median (IQR) & $15.7(6.4-18.5)$ & $7.8(4.8-11.4)$ & \\
\hline \multicolumn{4}{|l|}{ Initial platelets } \\
\hline Median (IQR) & $149(100-280)$ & $268(203-392)$ & 0.0025 \\
\hline
\end{tabular}

Organ dysfunction defined as pSOFA $>=2$, No organ dysfunction included floor patients and ICU patients with pSOFA $<2$

a Patients $<2$ years old excluded $(n=25)$ and $n=7$ missing $>=2$ years old

b Ferritin collected for 42 patients

c CRP collected for 37 patients

Limitations of our study include its retrospective design and relatively small sample size due to this disease's emerging nature. Multivariable analysis was not possible due to limited sample size and missing data. Comparisons herein are intended to develop hypotheses for future testing. Increasing sample size could allow for 
further definition of risk factors associated with severe disease and organ dysfunction and allow for refinement of predictive factors of severe disease. Seven factors were identified a priori and to maintain appropriate power and study design; additional laboratory differences could not be evaluated for statistical significance between groups. Other statistical comparisons between groups of patients could be an area of future research.

There were missing lab values, more prominently in the general floor cohort with a shorter LOS and lower disease severity. This skew in missing data is typical of retrospective studies, given healthier patients tend to have less clinical indication for laboratory studies. Despite this, the data suggests an association between elevations in the inflammatory markers discussed and presence of organ dysfunction. CRP, WBC, and thrombocytopenia may be useful tools in predicting patients who will develop organ dysfunction and is a vital area of future research. As the COVID-19 pandemic has overwhelmed healthcare systems, early markers of organ dysfunction could assist in triage decisions. Future studies will be able to include a larger cohort of patients and allow for refined study of the clinical characteristics of severe pediatric COVID-19.

\section{Conclusions}

COVID-19 can cause severe, life-threatening disease in children. Older children are more likely to require PICU admission. Our cohort did not find an association between underlying comorbidity and critical illness or organ dysfunction. CRP, WBC, and platelet count may be useful markers of organ dysfunction, and further study is needed.

\begin{abstract}
Abbreviations
BMI: Body mass index; CCMC: Cohen Children's Medical Center; COVID-19: Coronavirus disease-2019; CRP: C-reactive protein; HFNC: High-flow nasal cannula; ICU: Intensive Care Unit; IQR: Interquartile range; LMV: Length of mechanical ventilation; LOS: Length of stay; MIS-C: Multisystem Inflammatory Syndrome in Children; MV: Mechanical ventilation; NIPPV: Non-invasive positive pressure ventilation; OSI: Oxygen saturation index; pARDS: Pediatric acute respiratory distress syndrome; PELOD-2: Pediatric Logistic Organ Dysfunction-2; PICU: Pediatric intensive care unit; pSOFA: Pediatric Sequential Organ Failure Assessment; SARS-2-CoV: Severe acute respiratory syndrome coronavirus 2; SIUH: Staten Island University Hospital; SOFA: Sequential Organ Failure Assessment; SpO2: Oxygen saturation; US: United States; WBC: White blood cell.
\end{abstract}

\section{Acknowledgements}

We would like to acknowledge Dr. Megan Khariton and Dr. Pamela Feuer who contributed patient data as part of the Northwell COVID-19 research consortium.

\section{Authors' contributions}

GF, SH, CC, JS, TS, CS, and MT contributed to study design and manuscript preparation and review. SI contributed to statistical analysis and manuscript preparation and review. GF, SS, DL, MK, JB, LF, FM, and CK contributed to data abstraction and manuscript review.

\section{Authors' information}

GF and DL are Pediatric Critical Care Medicine (PCCM) fellows. MT, SS, CC, TS, and JS are PCCM attendings. MT performs NIH-funded research on immunity and sepsis. MK and SH are pediatric infectious diseases attendings. CS is the Chairman of Pediatrics who practiced PCCM.

\section{Funding}

Dr. Taylor receives NIGMS grant funding, \#K08GM132794. This work was supported by grants R24AG064191 from the National Institute on Aging of the National Institutes of Health and R01LM012836 from the National Library of Medicine of the National Institutes of Health. The views expressed in this paper are those of the authors and do not represent the views of the National Institutes of Health, the United States Department of Health and Human Services, or any other government entity.

\section{Availability of data and materials}

The data that support the findings of this study are available on request from cOVID19@northwell.edu.

\section{Ethics approval and consent}

The study was approved by the internal review board (\#20-0318-CCMC) and given a waiver of informed consent.

Consent for publication

Not applicable.

\section{Competing interests}

The authors have no competing interests to report.

\section{Author details}

${ }^{1}$ Division of Pediatric Critical Care Medicine, Steven and Alexandra Cohen Children's Medical Center of New York, Northwell Health, Donald and Barbara Zucker School of Medicine At Hofstra/Northwell, 269-01 76th Ave, New Hyde Park, NY 11040, USA. ${ }^{2}$ Department of Pediatrics, Steven and Alexandra Cohen Children's Medical Center of New York, Northwell Health, Donald and Barbara Zucker School of Medicine At Hofstra/Northwell, New Hyde Park, NY 11040, USA. ${ }^{3}$ Center for Health Innovations and Outcomes Research, Feinstein Institutes for Medical Research, Northwell Health, Manhasset, NY 11030, USA ${ }^{4}$ Division of Pediatric Infectious Diseases, Steven and Alexandra Cohen Children's Medical Center, Northwell Health, Donald and Barbara Zucker School of Medicine At Hofstra/Northwell, New Hyde Park, NY 11040, USA. ${ }^{5}$ Division of Pulmonary, Critical Care, and Sleep Medicine, Northwell Health, Donald and Barbara Zucker School of Medicine At Hofstra/Northwell, New Hyde Park, NY 11040, USA. ${ }^{6}$ Division of Pediatric Surgery, Steven and Alexandra Cohen Children's Medical Center of New York, Northwell Health, Donald and Barbara Zucker School of Medicine At Hofstra/Northwell, New Hyde Park, NY 11040, USA.

Received: 10 September 2020 Accepted: 10 December 2020 Published online: 19 December 2020

\section{References}

1. Dong E, Du H, Garnder L. An interactive web-based dashboard to track COVID-19 in real time. Lancet Infect Dis. 2020;20(5):533-4. https://doi. org/10.1016/S1473-3099(20)30120-1.

2. Xie J, Tong Z, Guan X, Du B, Qiu H, Slutsky AS. Critical care crisis and some recommendations during the COVID-19 epidemic in China. Intensive Care Med. 2020;46(5):837-40. https://doi.org/10.1007/s00134-020-05979 $-7$.

3. Dong Y, Mo X, Hu Y, Qi X, Jiang F, Jiang Z, et al. Epidemiological characteristics of 2143 pediatric patients with 2019 coronavirus disease in China. Pediatrics. 2020;145(6):e20200702. https://doi.org/10.1542/ peds.2020-0702.

4. Cai J, Xu J, Lin D, Yang Z, Xu L, Qu Z, et al. A Case Series of children with 2019 novel coronavirus infection: clinical and epidemiological features. Clin Infect Dis. 2020;71(6):1547-51. https://doi.org/10.1093/cid/ciaa198.

5. Sun D, Li H, Lu XX, Xiao H, Ren J, Zhang FR, et al. Clinical features of severe pediatric patients with coronavirus disease 2019 in Wuhan: a single center's observational study. World J Pediatr. 2020. 
6. Zheng F, Liao C, Fan QH, Chen HB, Zhao XG, Xie ZG, et al. Clinical charac teristics of children with Coronavirus Disease 2019 in Hubei, China. Curr Med Sci. 2020;40:275-80. https://doi.org/10.1007/s11596-020-2172-6.

7. Lu X, Zhang L, Du H, Zhang J, Li YY, Qu J, et al. SARS-CoV-2 infection in children. N Engl J Med. 2020;382(17):1663-5. https://doi.org/10.1056/ NEJMc2005073.

8. Song W, Li J, Zou N, Guan W, Pan J, Xu W. Clinical features of pediatric patients with coronavirus disease (COVID-19). J Clin Virol. 2020:127:104377.

9. Dong Y, Mo X, Hu Y, Qi X, Jiang F, Jiang Z, et al. Epidemiology of COVID-19 among children in China. Pediatrics. 2020;145(6).

10. Parri N, Lenge $M$, Buonsenso D, Coronavirus Infection in pediatric emergency departments research $\mathrm{G}$. children with Covid-19 in pediatric emergency departments in Italy. N Engl J Med. 2020.

11. Shekerdemian L, Mahmood N, Wolfe K, Riggs B, Ross C, McKiernan C, et al Characteristics and outcomes of children with Coronavirus Disease 2019 (COVID-19) infection admitted to us and Canadian pediatric intensive care units. JAMA Pediatr. 2020;174(9):868-73. https://doi.org/10.1001/ jamapediatrics.2020.1948.

12. Derespina KR, Kaushik S, Plichta A, Conway EE Jr, Bercow A, Choi J, et al. Clinical manifestations and outcomes of critically III children and adolescents with COVID-19 in New York City. J Pediatr. 2020;226(55.e2):63.e2. https://doi.org/10.1016/j.jpeds.2020.07.039

13. Zachariah $\mathrm{P}$, Johnson $\mathrm{CL}$, Halabi KC, Ahn D, Sen Al, Fischer A, et al. Epidemiology, clinical features, and disease severity in patients with Coronavirus disease 2019 (COVID-19) in a children's hospital in New York City, New York. JAMA Pediatr. 2020;174(10):e202430. https://doi.org/10.1001/ jamapediatrics.2020.2430.

14. Yu Y, Chen P. Coronavirus disease 2019 (COVID-19) in neonates and children from China: a review. Front Pediatr. 2020;8:287.

15. Zimmermann $P$, Curtis N. Coronavirus infections in children including COVID-19: an overview of the epidemiology, clinical features, diagnosis, treatment and prevention options in children. Pediatr Infect Dis J. 2020;39(5):355-68. https://doi.org/10.1097//NF.0000000000002660.

16. She J, Liu L, Liu W. COVID-19 epidemic: disease characteristics in children. J Med Virol. 2020;92(7):747-54

17. Kainth MK, Goenka PK, Williamson KA, Fishbein JS, Subramony A, Schleien C, et al. Early experience of COVID-19 in a US Children' Hospital. Pediatrics. 2020;146(4):e2020003186. https://doi.org/10.1542/peds.2020-003186.

18. Matics TJ, Sanchez-Pinto LN. Adaptation and validation of a pediatric sequential organ failure assessment score and evaluation of the sepsis-3 definitions in critically III children. JAMA Pediatr. 2017:171(10):e172352.

19. Leteurtre S, Duhamel A, Salleron J, Grandbastien B, Lacroix J, Leclerc F, et al. PELOD-2: an update of the PEdiatric logistic organ dysfunction score. Crit Care Med. 2013;41(7):1761-73.
20. Pediatric Acute Lung Injury Consensus Conference G. Pediatric acute respiratory distress syndrome: consensus recommendations from the Pediatric Acute Lung Injury Consensus Conference. Pediatr Crit Care Med. 2015;16(5):428-39.

21. Harris PA, Taylor R, Thielke R, Payne J, Gonzalez N, Conde JG. Research electronic data capture (REDCap)-a metadata-driven methodology and workflow process for providing translational research informatics support. J Biomed Inform. 2009;42(2):377-81.

22. Harris PA, Taylor R, Minor BL, Elliott $V$, Fernandez M, O'Neal L, et al. The REDCap consortium: building an international community of software platform partners. J Biomed Inform. 2019;95:103208.

23. Ruan Q, Yang K, Wang W, Jiang L, Song J. Clinical predictors of mortality due to COVID-19 based on an analysis of data of 150 patients from Wuhan, China. Intensive Care Med. 2020;46(5):846-8. https://doi. org/10.1007/s00134-020-05991-x

24. Carcillo JA, Sward K, Halstead ES, Telford R, Jimenez-Bacardi A, Shakoory $B$, et al. A systemic inflammation mortality risk assessment contingency table for severe sepsis. Pediatr Crit Care Med. 2017;18(2):143-50.

25. Garg SKL, Whitaker M, et al. Hospitalization rates and characteristics of patients hospitalized with laboratory-confirmed Coronavirus Disease 2019-COVID-NET, 14 States, March 1-30, 2020. MMWR Morb Mortal Wkly Rep [Internet]. 2020;69(15):458-64.

26. A SAS program for the 2000 CDC growth charts (ages 0 to $<20$ years) centers for disease control and prevention. https://www.cdc.gov/nccdp hp/dnpao/growthcharts/resources/sas.htm.

27. Weir CB, Jan A. BMI classification percentile and cut off points. In: StatPearls. StatPearls Publishing: Treasure Island (FL); 2020.

28. Vincent JL, Moreno R, Takala J, Willatts S, De Mendonca A, Bruining $\mathrm{H}$, et al. The SOFA (Sepsis-related Organ Failure Assessment) score to describe organ dysfunction/failure On behalf of the Working Group on Sepsis-Related Problems of the European Society of Intensive Care Medicine. Intensive Care Med. 1996;22(7):707-10.

29. Singer $M$, Deutschman CS, Seymour CW, Shankar-Hari M, Annane D, Bauer $M$, et al. The third international consensus definitions for sepsis and septic shock (Sepsis-3). JAMA. 2016;315(8):801-10.

30. Ogimi C, Englund JA, Bradford MC, Qin X, Boeckh M, Waghmare A. Characteristics and outcomes of coronavirus infection in children: the role of viral factors and an immunocompromised state. J Pediatric Infect Dis Soc. 2019;8(1):21-8

\section{Publisher's Note}

Springer Nature remains neutral with regard to jurisdictional claims in published maps and institutional affiliations.

\section{Submit your manuscript to a SpringerOpen ${ }^{\circ}$ journal and benefit from:}

- Convenient online submission

- Rigorous peer review

- Open access: articles freely available online

- High visibility within the field

Retaining the copyright to your article

Submit your next manuscript at springeropen.com 